\title{
FEM-BEM Coupling for the MHD Pipe Flow in an Exterior Region
}

\author{
S. Han Aydin \\ Department of Mathematics, Karadeniz Technical University, Trabzon, Turkey \\ Email: shaydin@ktu.edu.tr
}

Received June 11, 2013; revised July 10, 2013; accepted July 24, 2013

Copyright (C) 2013 S. Han Aydın. This is an open access article distributed under the Creative Commons Attribution License, which permits unrestricted use, distribution, and reproduction in any medium, provided the original work is properly cited.

\begin{abstract}
In this study, the magnetohydrodynamic (MHD) flow through a circular pipe under the influence of a transverse magnetic field when the outside medium is also electrically conducting is solved numerically by using FEM-BEM coupling approach. The coupled partial differential equations defined for the interior medium are transformed into homogenous modified Helmholtz equations. For the exterior medium on an infinite region, the Laplace equation is considered for the exterior magnetic field. Unknowns in the equations are also related with the corresponding Dirichlet and Neumann type coupled boundary conditions. Unknown values of the magnetic field on the boundary and for the exterior region are obtained by using BEM, and the unknown velocity and magnetic field inside the pipe are obtained by using SUPG type stabilized FEM. Computations are carried for very high values of magnetic Reynolds numbers $R m_{1}$, Reynolds number $R e$ and magnetic pressure $R h$ of the fluid. The results show that using stabilized method enables us to get stable and accurate numerical approximations consistent with the physical configuration of the problem over rough mesh which also results a cheap computational cost.
\end{abstract}

Keywords: FEM-BEM Coupling; SUPG; MHD Pipe Flow

\section{Introduction}

The objective of this work is to present MHD pipe flow of circular cross-section under the influence of a transverse magnetic field numerically by using FEM-BEM coupling approach when the outside medium is also electrically conducting. It is already known that there are many applications of MHD pipe flow such as the design of the cooling systems with liquid metals for nuclear reactors, electromagnetic pumps, MHD generators, and flowmeters measuring blood pressure, etc. The exact solution of the problem can be obtained only for some special cases $[1,2]$. Therefore, there are many numerical methods applied to the solution of the MHD pipe flow (see [3-9] and references there in).

The boundary element method is a technique which offers a great advantage to analyze stationary problems especially with infinite domains [10]. BEM has the advantage of discretizing only the pipe wall and gives the solution both in the exterior and interior regions at any point. Due to the regularity conditions, it is possible to limit the discretization to a finite boundary.

The finite element method is one of the most preferable numerical method applied to the solution of many physical and engineering problems. Applications of the finite element method to MHD problems are already performed by many researchers [11-14]. But, in standard Galerkin formulations, there exist numerical instabilities for the high Hartmann number value cases. It is possible to eliminate these, either using finer mesh which increases the computational cost, or considering stabilized finite element methods [15-18]. One of the most popular of such numerical methods is referred as the streamline upwind Petrov-Galerkin (SUPG) method [19]. The finite element method of the SUPG type reduces the oscillations in the standard Galerkin method and achieves stability by adding mesh-dependent perturbation terms to the formulation. These terms enhance the coercivity of the formulation by acting like artificial diffusion in the direction of the flow.

The present paper deals with the solution of the general MHD problem inside of a pipe when the surrounding medium is also electrically conducting, and has small magnetization compared to the fluid inside the pipe. The flow is assumed as incompressible and viscous, and the fluid inside the pipe is electrically conducting. The outside medium has also electrical conductivity which 
is small compared to fluid. However, the continuity of both induced magnetic fields inside and outside of the pipe is maintained through the conducting wall of the pipe. Therefore, this problem is a generalization of the special cases of MHD flow through a pipe. Thus, we consider in this paper, the most general case of MHD flow in a pipe which has electrically conducting wall with the same conductivity of outside infinite medium. This way, partial differential equations defined inside the pipe and outside infinite region are solved simultaneously with the coupled induced current conditions on the pipe wall. Equations defined inside the pipe are decoupled and transformed to homogenous modified Helmholtz equations for which the BEM formulations are given with fundamental solution $K_{0}(x)$. BEM formulation in the exterior region is carried with the logarithmic fundamental solution of Laplace equation. Using this formulation, unknown magnetic field values are obtained on the boundary of the pipe.

The paper is organized as follows: In Section 2, we describe the mathematical modelling of the equations. BEM and FEM coupling numerical solution procedure is presented in Section 3. Application of the prescribed algorithm is discussed in Section 4 by presenting some numerical experiments obtained from different values of magnetic Reynolds numbers $R m_{1}$, Reynolds number $R e$ and magnetic pressure $R h$ of the fluid. Conclusions are drawn in Section 5.

\section{Mathematical Model}

The MHD flow is governed by a set of coupled PDE's obtained from Navier-Stokes equations for conducting fluids, and Maxwell's equations for electromagnetic field through Ohm's law. We consider MHD flow in a straight pipe (channel, duct) of sufficient length, and of crosssection a circle in the $x y$-plane. The fluid is flowing through the pipe due to an applied constant pressure gradient $\frac{\partial p}{\partial z}$, and is viscous, incompressible, electrically conducting. The electrical permitivity and magnetic permeability of the fluid are assumed to be close to those of the external space. The axis of the pipe is coincident with the $z$-axis, and the $y$-axis is parallel with the magnetic induction at infinity. Thus, externally applied magnetic field with an intensity $B_{0}$ is assumed to be in $y$ direction. It determines the appearance within the fluid of an induced magnetic field (induced current). We assume that the wall of the pipe and the outside medium are also electrically conducting having the same electrical conductivity and magnetic permeability since the thickness of pipe wall is assumed to be very small (Figure 1).

Thus, the non-dimensional form of the equations to be solved inside the pipe and its exterior are $[20,21]$



Figure 1. Problem definition (left) and discretization of the domain (pipe and external region) (left).

$$
\begin{aligned}
& \nabla^{2} V(x, y)+R e R h \frac{\partial B}{\partial y}(x, y)=-1 \\
& \nabla^{2} B(x, y)+R m_{1} \frac{\partial V}{\partial y}(x, y)=0 \\
& \nabla^{2} B_{\text {ext }}(x, y)=0 \quad \text { in } \Omega_{\text {ext }}
\end{aligned}
$$

with the no-slip condition on the pipe wall

$$
V=0 \quad \text { on } \partial \Omega_{\text {in }}=\Gamma
$$

and continuity conditions for the induced magnetic fields

$$
\begin{gathered}
B(x, y)=B_{e x t}(x, y), \quad \text { on } \Gamma \\
\frac{1}{R m_{1}} \frac{\partial B(x, y)}{\partial n}=\frac{1}{R m_{2}} \frac{\partial B_{e x t}(x, y)}{\partial n^{\prime}}, \text { on } \Gamma \\
\lim _{x^{2}+y^{2} \rightarrow \infty} B_{e x t}(x, y)=0
\end{gathered}
$$

where $n$ and $n^{\prime}$ are unit outward normals on $\Gamma$ for the regions $\Omega_{\text {in }}$ and $\Omega_{\text {ext }}$, respectively. $R m_{1}$ and $R m_{2}$ are the magnetic Reynolds numbers inside the pipe and in external medium.

MHD Equation (1) are decoupled first by denoting

$V_{1}=V$ and $B_{1}=\frac{R e R h}{M} B$, where $M=\sqrt{R e R h R m_{1}}$ is the Hartmann number of the fluid, the system (1) becomes $[1,20]$

$$
\begin{aligned}
& \nabla^{2} V_{1}+M \frac{\partial B_{1}}{\partial y}=-1 \\
& \nabla^{2} B_{1}+M \frac{\partial V_{1}}{\partial y}=0 .
\end{aligned}
$$

As a further transformation, define new variables $U_{1}(x, y)$ and $U_{2}(x, y)$ as

$$
\begin{aligned}
& U_{1}=V_{1}+B_{1} \\
& U_{2}=V_{1}-B_{1}
\end{aligned}
$$

in order to decouple the Equations (7) as

$$
\begin{aligned}
& \nabla^{2} U_{1}+M \frac{\partial U_{1}}{\partial y}=-1 \\
& \nabla^{2} U_{2}-M \frac{\partial U_{2}}{\partial y}=-1 .
\end{aligned}
$$


For obtaining homogeneous equations, let's define $W_{1}(x, y)$ and $W_{2}(x, y)$

$$
\begin{aligned}
& W_{1}=U_{1}+\frac{1}{M} y \\
& W_{2}=U_{2}-\frac{1}{M} y
\end{aligned}
$$

which result in

$$
\begin{aligned}
& \nabla^{2} W_{1}+M \frac{\partial W_{1}}{\partial y}=0 \\
& \nabla^{2} W_{2}-M \frac{\partial W_{2}}{\partial y}=0 .
\end{aligned}
$$

Finally, the transformation with

$$
\begin{aligned}
& u_{1}=W_{1} \mathrm{e}^{k y} \\
& u_{2}=W_{2} \mathrm{e}^{-k y}
\end{aligned}
$$

where $k=M / 2$, reduces the equations to two homogeneous modified Helmholtz equations

$$
\begin{aligned}
& \nabla^{2} u_{1}-k^{2} u_{1}=0 \\
& \nabla^{2} u_{2}-k^{2} u_{2}=0 .
\end{aligned}
$$

Now, the system of Equations (1) and (2) are transformed to

$$
\begin{aligned}
& \nabla^{2} u_{1}-k^{2} u_{1}=0 \\
& \nabla^{2} u_{2}-k^{2} u_{2}=0 \\
& \nabla^{2} B_{e x t}=0 \quad \text { in } \Omega_{i n}
\end{aligned}
$$

with the boundary conditions (4)-(6) on $\Gamma$ which are also transformed in terms of new variables [21]

$$
\begin{aligned}
& \mathrm{e}^{-k y} u_{1}+\mathrm{e}^{k y} u_{2}=0 \\
& \mathrm{e}^{-k y} u_{1}-\mathrm{e}^{k y} u_{2}=\frac{2 R e R h}{M} B_{e x t}+\frac{2}{M} y \\
& \mathrm{e}^{-k y} \frac{\partial u_{1}}{\partial n}-\mathrm{e}^{k y} \frac{\partial u_{2}}{\partial n}=\frac{2 M}{R m_{2}} \frac{\partial B_{e x t}}{\partial n^{\prime}}+\frac{2}{M} \frac{\partial y}{\partial n} .
\end{aligned}
$$

\section{FEM-BEM Coupling}

Using the Equations (14)-(15) with the boundary conditions (16)-(18), it is possible to obtain

$u_{1}, \frac{\partial u_{1}}{\partial n}, u_{2}, \frac{\partial u_{2}}{\partial n}, B_{e x t}, \frac{\partial B_{e x t}}{\partial n^{\prime}}$ on the boundary of the pipe wall using boundary element method only discretizing the boundary. After calculating these unknown values on the boundary, one can also able to obtain exterior values of $B_{\text {ext }}$ again using BEM. It is also possible to calculate the unknown values $V$ and $B$ at any point inside the pipe as done in [21].

However, for the large values of $R m_{1}, R e$ or $R h$ which increase the value of the Hartmann number $M$,
BEM solutions obtained using less number of discretization points brings some numerical instabilities because of the convection dominated behavior of the Equation (1). Therefore, these equations should be solved over coarse mesh using stabilization technique.

One of the well-known stabilization method is streamline upwind Petrov-Galerkin (SUPG) finite element method [19] which is applicable for the convection dominated case of the convection diffusion type equations.

In order to apply SUPG stabilization, transformed form of the equations

$$
\begin{aligned}
& \nabla^{2} U_{1}+M \frac{\partial U_{1}}{\partial y}=-1 \\
& \nabla^{2} U_{2}-M \frac{\partial U_{2}}{\partial y}=-1
\end{aligned}
$$

with the boundary conditions

$$
U_{1}=B_{1} \text { and } U_{2}=-B_{1} \text { on } \Gamma
$$

are used. The weak formulation of the problem (9) can be stated as [22]: Find $U_{1}, U_{2} \in V=H_{0}^{1}(\Omega)^{2}$ such that

$$
\left.\begin{array}{l}
\left(\nabla U_{1}, \nabla v\right)-M\left(\frac{\partial U_{1}}{\partial y}, v\right)=(1, v) \\
\left(\nabla U_{2}, \nabla v\right)+M\left(\frac{\partial U_{2}}{\partial y}, v\right)=(1, v)
\end{array}\right\}, \quad \forall v \in V .
$$

To introduce a finite element method, we begin by partitioning the domain into triangular elements in a standard way (e.g. no overlapping, no vertex on the edge of a neighboring elements, etc.) and let $\Omega_{h}$ be such a partition of $\Omega$ and a finite dimensional space $V_{h}$ which is related to the choice of partition and satisfies $V_{h} \subset V$. Now the Galerkin finite element formulation of the problems reads; find $U_{1 h}, U_{2 h} \in V_{h}$ such that

$$
\left.\begin{array}{l}
\left(\nabla U_{1 h}, \nabla v_{h}\right)-M\left(\frac{\partial U_{2 h}}{\partial y}, v_{h}\right)=\left(1, v_{h}\right) \\
\left(\nabla U_{2 h}, \nabla v_{h}\right)+M\left(\frac{\partial U_{2 h}}{\partial y}, v_{h}\right)=\left(1, v_{h}\right)
\end{array}\right\}, \forall v_{h} \in V_{h} .
$$

Finally, if we insert the stabilization terms to the formulation, the SUPG type stabilized FEM variational formulation is written as [18,19]: find $U_{1 h}, U_{2 h} \in V_{h}$ such that

$$
\begin{aligned}
& {\left[\begin{array}{c}
\left(\nabla U_{1 h}, \nabla v_{h}\right)-M\left(\frac{\partial U_{2 h}}{\partial y}, v_{h}\right)-\left(1, v_{h}\right) \\
\left(\nabla U_{2 h}, \nabla v_{h}\right)+M\left(\frac{\partial U_{2 h}}{\partial y}, v_{h}\right)-\left(1, v_{h}\right)
\end{array}\right]} \\
& +\tau_{K}\left[\begin{array}{c}
\left(-M \frac{\partial U_{1 h}}{\partial y}-1\right)\left(-M \frac{\partial v_{h}}{\partial y}\right) \\
\left(M \frac{\partial U_{2 h}}{\partial y}-1\right)\left(M \frac{\partial v_{h}}{\partial y}\right)
\end{array}\right]=0
\end{aligned}
$$


$\forall v_{h} \in V_{h}$ with the stabilization parameter

$$
\tau_{K}= \begin{cases}\frac{h_{K}}{2 M} & \text { if } P e_{k} \geq 1 \\ \frac{h_{K}^{2}}{12} & \text { if } P e_{k}<1\end{cases}
$$

where $h_{K}$ is the diameter of the element $K$, $P e_{K}=\frac{M h_{K}}{6}$ is the Peclet number. It is seen from $t$ formulation that the SUPG method is computationally cheap and practically easy to implement. It is also very effective way of obtaining numerically stable solutions for convection dominated problems.

\section{Numerical Results}

We consider a long pipe of circle cross-section defined by $\left\{(x, y): x^{2}+y^{2} \leq 1\right\}$. The boundary of the circular region in $I R^{2}$ is discretized by using $N=64$ constant boundary elements on which the node is at the center. Also, in order to calculate unknown on internal and external region, 316 points (corresponding to 694 triangular elements) inside the pipe and 720 points (corresponding 1408 triangular elements) are used (Figure 1). Discretized system of linear equations obtained from BEM formulation in order to obtain unknown values at the boundary are solved by using the solver DGESV and equations obtained from FEM formulation for the unknown values inside the pipe region are solved by using the sparse solver UMFPACK which are LAPACK driver routines from Netlib library. The behaviors of the velocity of the fluid and inside and outside induced currents (induced magnetic fields) are visualized in terms of contour plots for very high values of magnetic Reynolds numbers $R m_{1}$, Reynolds number $R e$ and magnetic pressure number $R h$ of the fluid.

Before we present numerical results, let's define the notations; Labels "F-BEM", "BEM" and "Ref" refers to solutions FEM and BEM Coupling, BEM only and Reference solutions, respectively. As a Reference solution, we take the solution of the same problem over very fine mesh.

For the small values of the Hartmann number, both solution obtained either FEM-BEM Coupling or BEM only are almost equal. Therefore, only the high values of the $R m_{1}, R e$ and $R h=10$ are presented. From Figure 2, for the values $R m_{1}=1000, R m_{2}=1, R e=1$ and $R h=$ 10 , it is seen that, although induced magnetic field (induced current) solution are almost same and smooth, for the velocity values, there are some numerical instabilities in the solutions obtained from BEM only method which are source from the convection dominated behavior of the Equations (21). However, FEM-BEM coupling solution technique eliminates these disturbances and shows the well-known characteristic of the MHD flow that is the existence of the boundary layer formation as Hartmann number getting large.

Figure 3 shows equal velocity and induced current lines, respectively, for very large value of Reynolds number $R e=100$ when $R m_{1}=100, R m_{2}=1$ and $R h=10$. It seen from that, the effect of the stabilization in FEMBEM Coupling is also seen from the induced current solution additional to the velocity solutions. The similar behaviour is also seen for the high value of the magnetic pressure number $R h=100$ from Figure 4. The accuracy of the FEM-BEM Coupling approach to BEM only is also displayed in terms of the maximum values of the velocity and induced currents by comparing with the Reference solution (Table 1). It is known that, for large values of the parameters, the problem has unstable numerical results especially in maximum and minimum values. This is also seen from the table that BEM solutions give higher maximum values compared to reference solution.

\section{Conclusion}

We consider a FEM-BEM coupling approach for the approximate solution of the MHD flow through a circular pipe under the influence of a transverse magnetic field when the outside medium is also electrically conducting. Coupled equations with coupled boundary conditions are solved first on the boundary of the pipe by transforming the modified Helmholtz equations and Laplace equation. Then, velocity and induced magnetic field inside the pipe are calculated by considering SUPG typed stabilized finite element method, and induced magnetic on the external region is calculated with constant boundary ele-

Table 1. Comparison maximum values of the velocity and induced current.

\begin{tabular}{cccccccccc}
\hline & Parameters & & & Velocity & & \multicolumn{3}{c}{ Induced Current } \\
\hline$R m_{1}$ & $R e$ & $R h$ & F-BEM & BEM & Ref & F-BEM & BEM & Ref \\
\hline 1000 & 1 & 10 & 0.0089 & 0.0096 & 0.0091 & 0.078 & 0.091 & 0.085 \\
100 & 100 & 10 & 0.0022 & 0.0028 & 0.0016 & 0.0008 & 0.001 & 0.0009 \\
100 & 1 & 100 & 0.0052 & 0.0058 & 0.0052 & 0.0081 & 0.0091 & 0.0087 \\
\hline
\end{tabular}



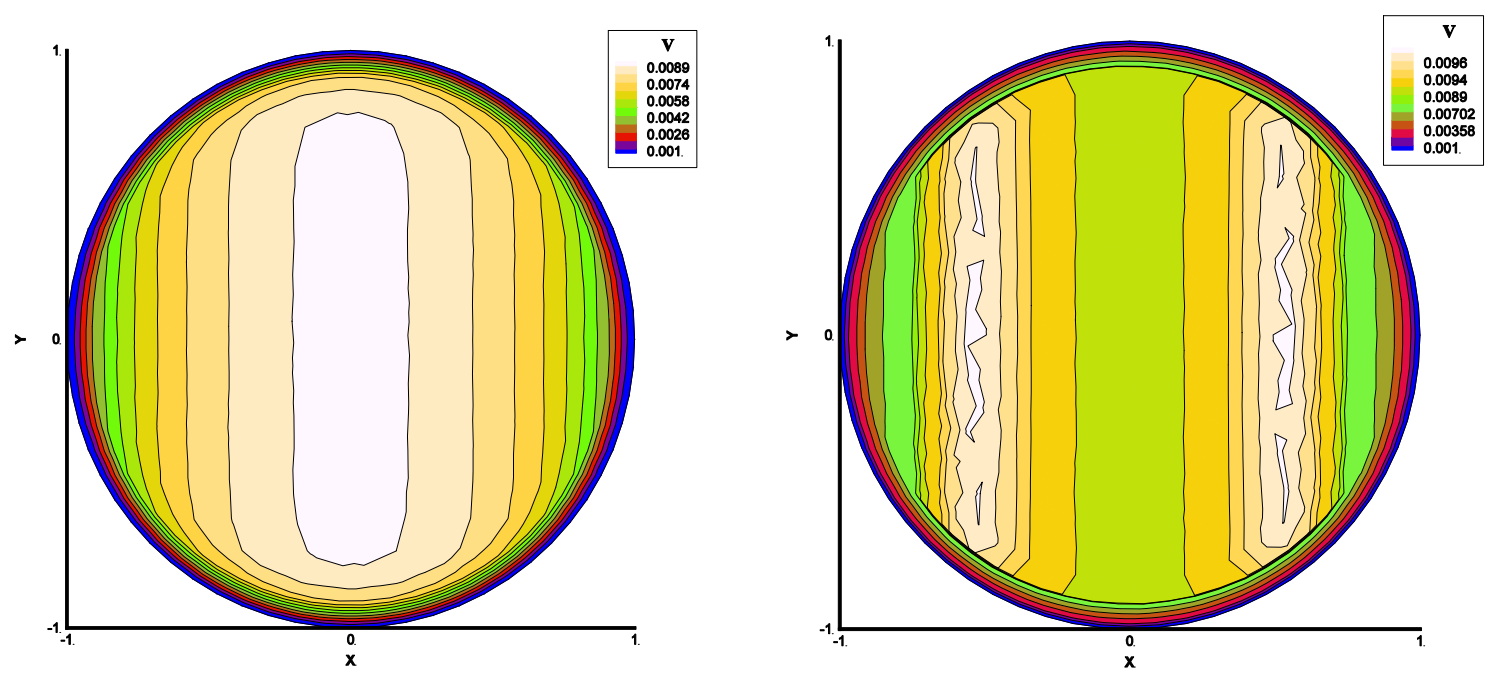

[Velocity]
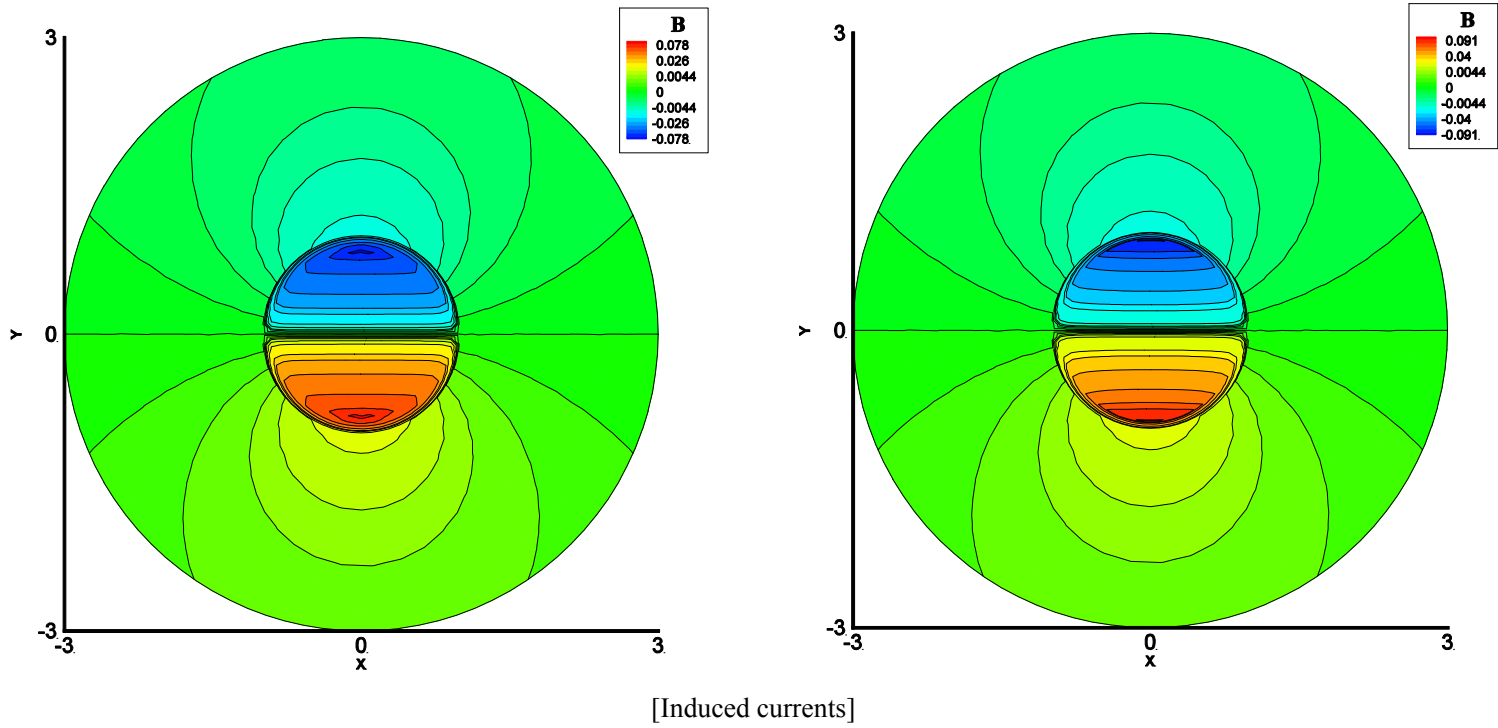

Figure 2. F-BEM (left) and BEM (right) contours for $R m_{1}=1000, R m_{2}=1, R e=1, R h=10$.
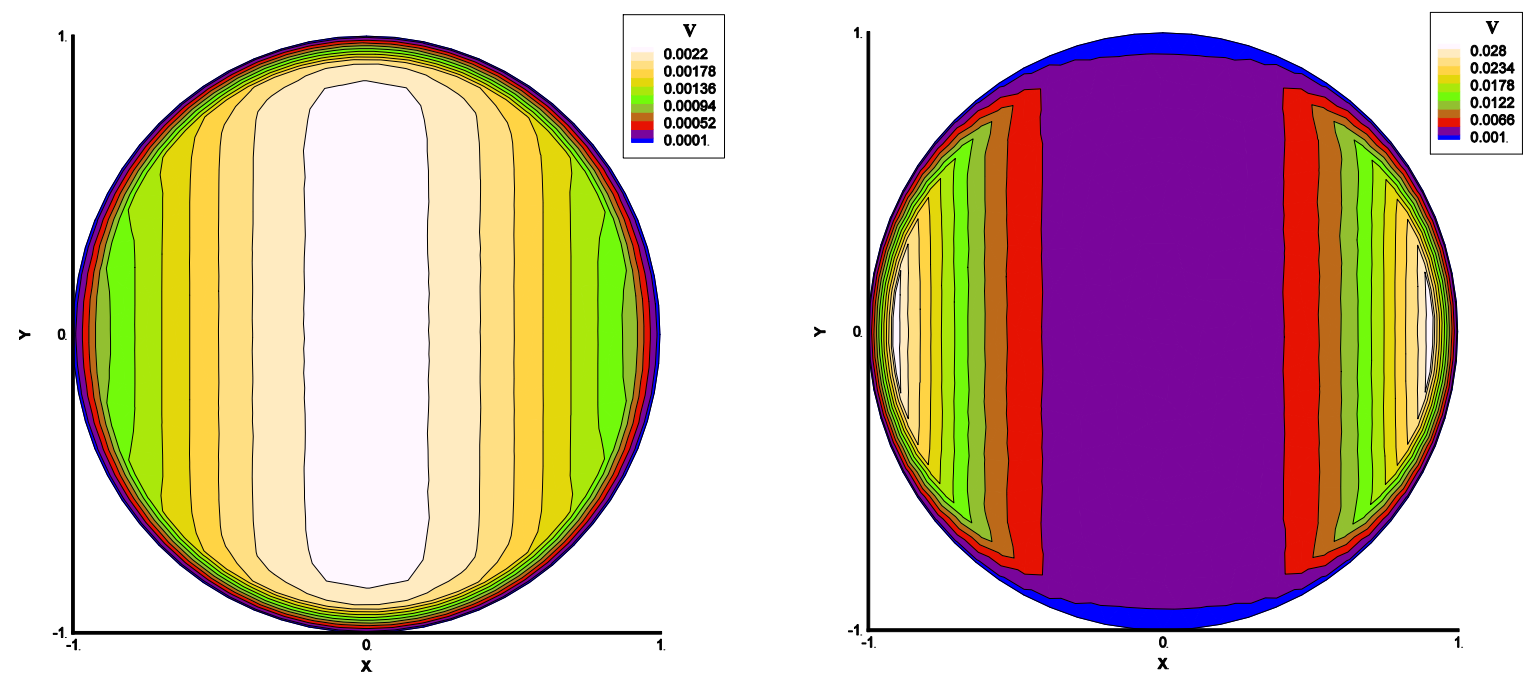

[Velocity] 


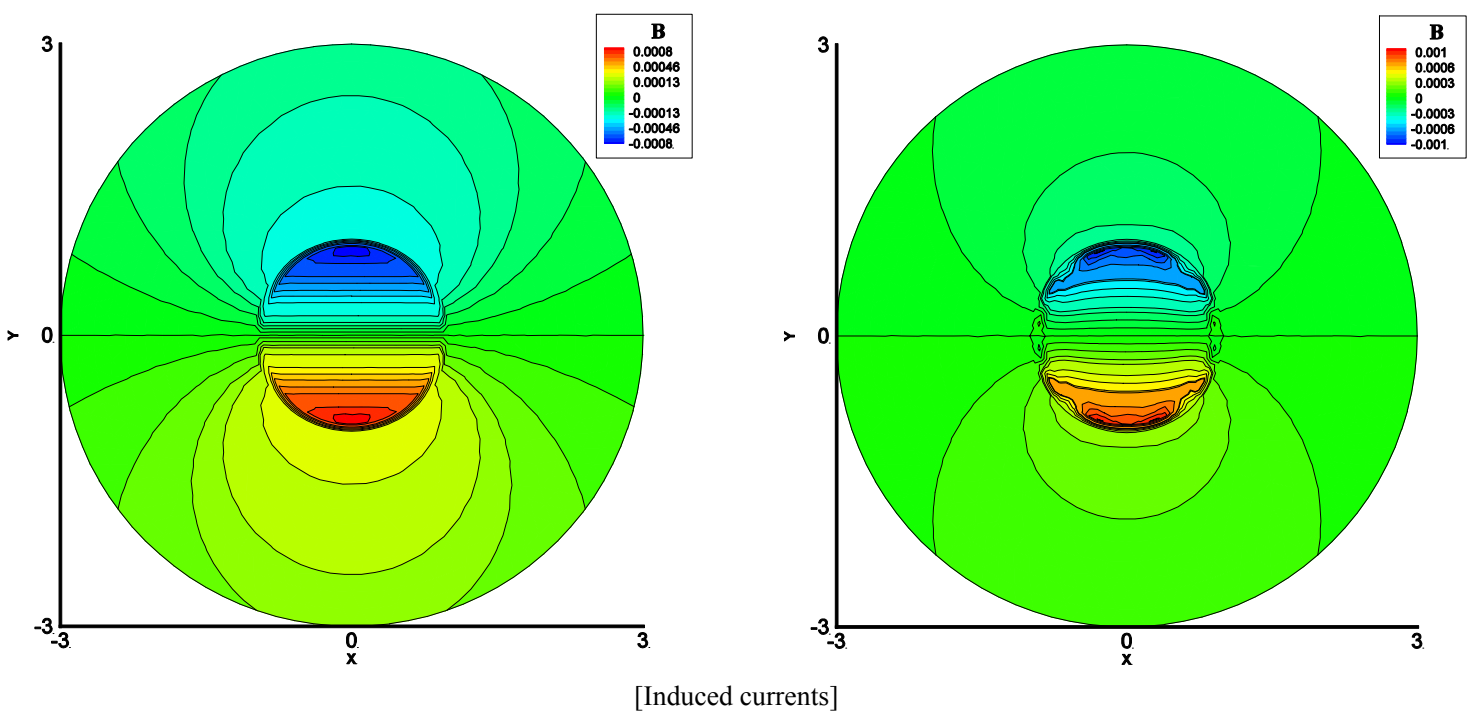

Figure 3. F-BEM (left) and BEM (right) contours for $R m_{1}=100, R m_{2}=1, R e=100, R h=10$.
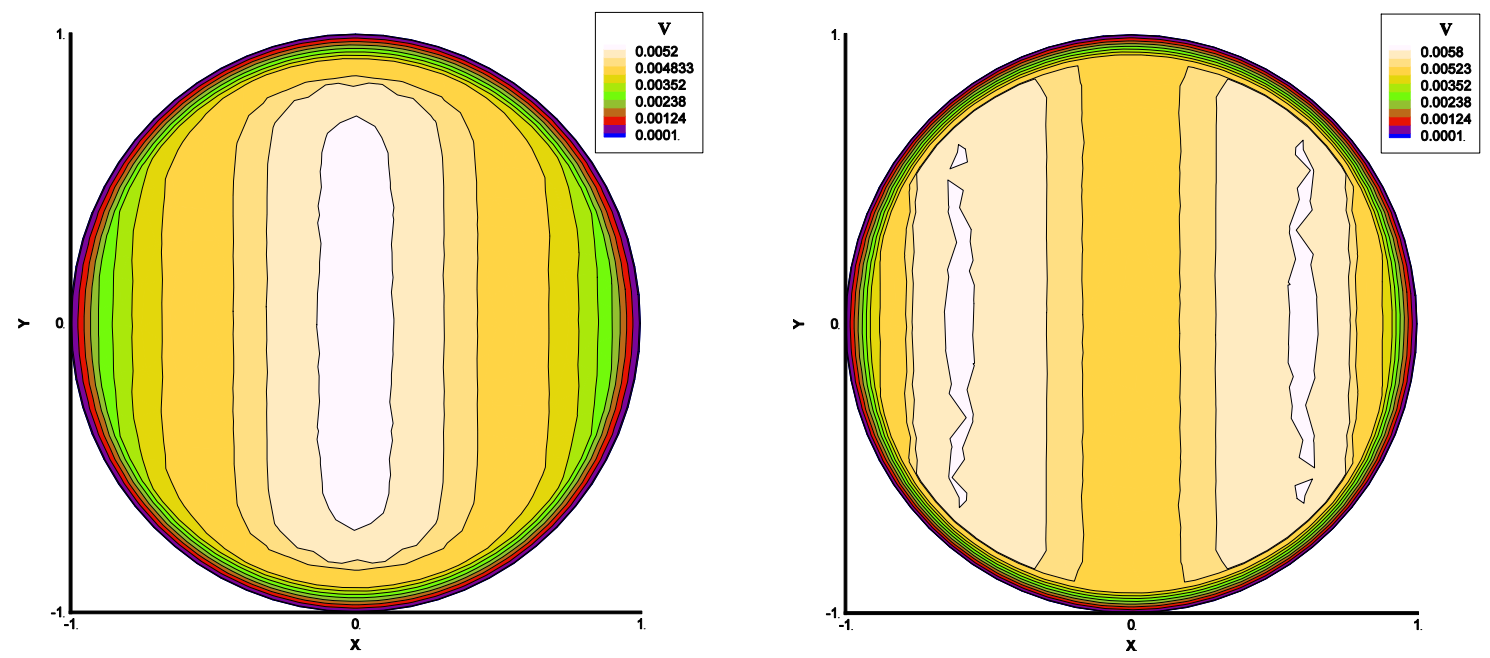

Figure 4. F-BEM (left) and BEM (right) velocity contours for $R m_{1}=100, R m_{2}=1, R e=1, R h=100$.

ment method due to the regularity conditions at infinity. Obtained solutions show the accuracy and efficiency of the proposed numerical scheme for the high values of the Hartmann number. Stabilized FEM-BEM Coupling approach is very effective not only in removing disturbances in numerical solutions but also in the maximum and minimum values of the unknowns.

\section{Acknowledgements}

This work is supported by Karadeniz Technical University by Scientific Research Project (BAP) under Grant Number \#10464.

\section{REFERENCES}

[1] L. Dragos, "Magnetofluid Dynamics," Abacus Press, Preston, 1975, pp. 92-99.
[2] J. A. Shercliff, "Steady Motion of Conducting Fluid in a Pipe under Transverse Magnetic Fields," Journal of Fluid Mechanics, Vol. 1, No. 6, 1956, pp. 644-666. doi:10.1017/S0022112056000421

[3] A. Carabineanu, A. Dinu and I. Oprea, "The Application of the Boundary Element Method to the Magnetohydrodynamic Duct Flow," Zeitschrift für Angewandte Mathematik und Physik, Vol. 46, No. 6, 1995, pp. 971-981. doi:10.1007/BF00917881

[4] T. W. H. Sheu and R. K. Lin, "Development of a Convection-Diffusion-Reaction Magnetohydrodynamic Solver on Nonstaggared Grids," International Journal for Numerical Methods in Fluids, Vol. 45, No. 11, 2004, pp. 12091233. doi:10.1002/fld. 738

[5] K. E. Barrett, "Duct Flow with a Transverse Magnetic Field at High Hartmann Numbers," International Journal for Numerical Methods in Engineering, Vol. 50, 2001, pp. 1893-1906. doi:10.1002/nme.101

[6] H. W. Liu and S. P. Zhu, "The Dual Reciprocity Boun- 
dary Element Method for Magnetohydrodynamic Channel Flows," ANZIAM Journal, Vol. 44, 2002, pp. 305-322. doi:10.1017/S1446181100013961

[7] M. Tezer-Sezgin and S. H. Aydn, "Dual Reciprocity Boundary Element Method for Magnetohydrodynamic Flow Using Radial Basis Functions," International Journal of Computational Fluid Dynamics, Vol. 16, No. 1, 2002, pp. 49-63. doi:10.1080/10618560290004026

[8] M. Tezer-Sezgin, "Boundary Element Method Solution of MHD Flow in Rectangular Duct," International Journal for Numerical Methods in Fluids, Vol. 18, No. 10, 1994, pp. 937-952. doi:10.1002/fld.1650181004

[9] M. Dehghan and D. Mirzai, "Meshless Local Boundary Integral Equation (LBIE) Method for the Unsteady Magnetohydrodynamic (MHD) Flow in Rectangular and Circular Pipes," Computer Physics Communications, Vol. 180, No. 9, 2009, pp. 1458-1466. doi:10.1016/j.cpc.2009.03.007

[10] F. Paris and J. Canas, "Boundary Element Method, Fundamentals and Applications," Oxford University Press Inc., New York, 1997, pp. 64-78.

[11] A. J. Meir, "Finite Element Analysis of Magnetohydrodynamic Pipe Flow," Applied Mathematics and Computation, Vol. 57, No. 2-3, 1993, pp. 177-196. doi:10.1016/0096-3003(93)90145-5

[12] M. Tezer-Sezgin and S. Koksal, "FEM for Solving MHD Flow in a Rectangular Duct," International Journal for Numerical Methods in Engineering, Vol. 28, No. 2, 1989, pp. 445-459. doi:10.1002/nme.1620280213

[13] N. B. Salah, A. Soulaimani, W. G. Habash and M. Fortin, "A Conservative Stabilized Finite Element Method for the Magneto-Hydrodynamic Equations," International Journal for Numerical Methods in Fluids, Vol. 29, No. 5, 1999 , pp. 535-554. doi:10.1002/(SICI)1097-0363(19990315)29:5<535::AIDFLD799>3.0.CO;2-D

[14] N. B. Salah, A. Soulaimani and W. G. Habashi, "A Finite Element Method for Magnetohydrodynamics," Computer
Methods in Applied Mechanics and Engineering, Vol. 190, No. 43-44, 2001, pp. 5867-5892. doi:10.1016/S0045-7825(01)00196-7

[15] A. I. Nesliturk and M. Tezer-Sezgin, "The Finite Element Method for MHD Flow at High Hartmann Numbers", Computer Methods in Applied Mechanics and Engineering, Vol. 194, No. 9-11, 2005, pp. 1201-1224. doi:10.1016/j.cma.2004.06.035

[16] J. F. Gerbeau, "A Stabilized Finite Element Method for the Incompressible Magnetohydrodynamic Equations," Numerische Mathematik, Vol. 87, No. 1, 2000, pp. 83111. doi: $10.1007 / \mathrm{s} 002110000193$

[17] R. Codina and N. H. Silva, "Stabilized Finite Element Approximation of the Stationary Magnetohydrodynamics Equations," Computational Mechanics, Vol. 38, No. 4-5, 2006, pp. 344-355. doi:10.1007/s00466-006-0037-x

[18] S. H. Aydin, A. I. Nesliturk and M. Tezer-Sezgin, "TwoLevel Finite Element Method with a Stabilizing Subgrid for the Incompressible MHD Equations," International Journal for Numerical Methods in Fluids, Vol. 62, No. 2, 2010, pp. 188-210.

[19] A. N. Brook and T. J. R. Hughes, "Streamline Upwind/ Petrov-Galerkin Formulations for Convection Dominated Flows with Particular Emphasis on the İncompressible Navier-Stokes Equations," Computer Methods in Applied Mechanics and Engineering, Vol. 32, No. 1-3, 1982, pp. 199-259. doi:10.1016/0045-7825(82)90071-8

[20] A. Carabineanu and E. Lungu, "Pseudospectral Method for MHD Pipe Flow," International Journal for $\mathrm{Nu}$ merical Methods in Engineering, Vol. 68, No. 2, 2006, pp. 173-191. doi:10.1002/nme.1706

[21] M. Tezer-Sezgin and S. H. Aydn, "BEM Solution of MHD Flow in a Pipe Coupled with Magnetic İnduction of Exterior Region," Computing, Vol. 95, No. 1, 2013, pp. 751770. doi:10.1007/s00607-012-0270-4

[22] J. N. Reddy, "An Introduction to the Finite Element Method," McGraw-Hill, New York, 1993. 